DESY 10-111

LMU-ASC 55/10

\title{
Proton Hexality in Local Grand Unification
}

\author{
Stefan Förste ${ }^{a}$, Hans Peter Nilles ${ }^{a}$, Saúl Ramos-Sánchez ${ }^{b}$ \\ and Patrick K.S. Vaudrevange ${ }^{c}$ \\ ${ }^{a}$ Bethe Center for Theoretical Physics and \\ Physikalisches Institut der Universität Bonn, \\ Nussallee 12, 53115 Bonn, Germany \\ ${ }^{b}$ Deutsches Elektronen-Synchrotron DESY, Hamburg, Germany \\ c Arnold Sommerfeld Center for Theoretical Physics, \\ Ludwig-Maximilians-Universität München, 80333 München, Germany
}

\begin{abstract}
Proton hexality is a discrete symmetry that avoids the problem of too fast proton decay in the supersymmetric extension of the standard model. Unfortunately it is inconsistent with conventional grand unification. We show that proton hexality can be incorporated in the scheme of "Local Grand Unification" discussed in the framework of model building in (heterotic) string theory.
\end{abstract}




\section{Introduction}

The question of proton stability is of great importance in elementary particle physics. Conserved baryon number (B) could be a reason for a stable proton but would be incompatible with the creation of a baryon asymmetry in the universe. In the standard $\mathrm{SU}(3) \times \mathrm{SU}(2) \times \mathrm{U}(1)$ model of particle physics $\mathrm{U}(1)_{B}$ is a good symmetry at the renormalisable level (broken by non-perturbative effects and possibly dimension- 6 operators) and proton decay is sufficiently suppressed. The phenomenon of B-violation is thus mainly concerned with physics beyond the standard model (SM). Indeed, a natural framework to address the question is grand unification (GUTs). Quarks and leptons appear in unified multiplets and $\mathrm{U}(1)_{B}$ is broken. The known stability of the proton requires the GUT scale to be rather large $M_{G U T}>10^{16} \mathrm{GeV}$ such that dimension-6 operators are sufficiently suppressed.

Over the years it appears that the framework of GUTs seems to require supersymmetry. This is then consistent with unification of gauge coupling constants at $M_{G U T} \sim 10^{16}$ but it leads to new complications with proton stability due to potentially dangerous dimension-4 and dimension-5 operators. New symmetries like R-parity [1] (or matter parity [2,3]) have been conjectured to forbid the dim- 4 operators. Such a symmetry would lead to a new stable particle as the source for dark matter. Still, the dim- 5 operators are problematic. Other discrete symmetries like baryon triality $\left(B_{3}\right)[4,5]$ might solve the problem. The most attractive symmetry is proton hexality $\left(P_{6}\right)$ and has been identified by Dreiner, Luhn and Thormeier [6]. It forbids all the problematic dim- 4 and -5 operators, but allows lepton number violation in form of Majorana neutrino masses: thus $P_{6}$ perfectly fits our needs.

Proton hexality is a beautiful symmetry and grand unification is a very attractive scheme: but unfortunately there is a clash between the two. $P_{6}$ is incompatible with a unified structure of quark and lepton multiplets [7]. This is in contrast to matter parity $\mathbb{Z}_{2}^{\text {matter }}$ which can be incorporated e.g. in an $\mathrm{SO}(10)$ GUT (it is a discrete subgroup of $\mathrm{U}(1)_{B-L}$ in $\left.\mathrm{SO}(10)\right)$. This is not true for $B_{3}$ and $P_{6}$. An ultraviolet (UV) completion of theories with $P_{6}$ needs something more general than grand unification. String theory could be a candidate as it also includes a consistent description of gravitational interactions 1

Recent work towards string theoretic constructions of the minimal supersymmetric standard model (MSSM) [8-12] has revealed the concept of "Local Grand Unification" [8, 13, 14, a variant of GUTs that addresses some of its problematic properties. It allows "split multiplets" that e.g. solve the doublet-triplet splitting in the Higgs-sector and simplifies the breakdown of the grand unified gauge groups. These incomplete or split multiplets make it possible that $P_{6}$ can become compatible with local grand unification.

Moreover, it has recently been argued that discrete symmetries appear abundantly in string model constructions [15-17], with important applications for particle physics model building [18 22]. We are thus in a situation that $P_{6}$ could originate from a string model as consistent UV completion and that such a symmetry is compatible with local grand

\footnotetext{
${ }^{1}$ Global symmetries might be broken by gravitational interactions. Therefore it is important to discuss these questions in theories where gravity is consistently incorporated.
} 
unification. Within this top-down approach we can be confident that the global symmetries are respected by gravitational interactions. 2

The present paper is devoted to the study of proton hexality within the framework of local grand unification. In section 2 we shall present $P_{6}$ followed in section 3 by a discussion of the incompatibility of $P_{6}$ with GUTs. In section 4 we shall try to incorporate $P_{6}$ via a bottom-up approach in extra-dimensional GUT-like theories and stress the geometric aspects of local grand unification. Section 5 presents the results of heterotic orbifold constructions towards the incorporation of $P_{6}$. We provide some toy models where $P_{6}$ appears in various different ways, anomalous or non-anomalous. A completely satisfactory model has not been found yet. Section 6 will discuss possible lines of future research in the direction of explicit model building.

\section{Proton Hexality}

In this section we will motivate proton hexality as a discrete symmetry in the supersymmetric extension of the SM in somewhat more detail. For derivations, however, we will refer to original literature.

Ensuring sufficient stability of the proton in theories beyond the SM often provides nontrivial restrictions. New fields can give rise to baryon or lepton number violating couplings. In supersymmetric extensions of the SM, for instance, the most general superpotential respecting renormalisability and gauge invariance is

$$
\begin{aligned}
W= & h_{i j}^{E} L_{i} H_{d} \bar{E}_{j}+h_{i j}^{D} Q_{i} H_{d} \bar{D}_{j}+h_{i j}^{U} Q_{i} H_{u} \bar{U}_{j}+\mu H_{d} H_{u} \\
& +\lambda_{i j k} L_{i} L_{j} \bar{E}_{k}+\lambda_{i j k}^{\prime} L_{i} Q_{j} \bar{D}_{k}+\kappa_{i} L_{i} H_{u} \\
& +\lambda_{i j k}^{\prime \prime} \bar{U}_{i} \bar{D}_{j} \bar{D}_{k},
\end{aligned}
$$

where $i, j, k$ are family indices and gauge indices are suppressed. Terms in the first line encode Yukawa couplings needed for lepton and quark mass generation and the $\mu$-term contribution to the Higgs potential. Terms in the second and third line of (1) violate lepton and baryon number, respectively. The lepton or baryon number violating couplings $\left(\lambda, \lambda^{\prime}, \kappa\right.$ and $\left.\lambda^{\prime \prime}\right)$ can be forbidden by imposing an additional discrete symmetry such as $R$-parity or matter parity. Compared to $R$-parity, matter parity is a $\mathbb{Z}_{2}$ symmetry under which all constituents of a chiral multiplet carry the same charge, viz. matter multiplets are odd whereas Higgs multiplets are even.

Assuming that the supersymmetric extension of the SM originates from a more fundamental theory, such as a GUT, we also have to discuss effective non-renormalisable couplings. The superpotential can contain dangerous terms respecting all the symmetries including matter parity,

$$
\kappa_{i j k l}^{(1)} Q_{i} Q_{j} Q_{k} L_{l}+\kappa_{i j k l}^{(2)} \bar{U}_{i} \bar{U}_{j} \bar{D}_{k} \bar{E}_{l} .
$$

\footnotetext{
${ }^{2}$ Earlier attempts in a bottom-up approach [4 7] tried to solve this problem through the notion of "anomaly free discrete symmetries". In the top-down approach we do not have to worry about these constraints as long as we are dealing with a consistent string model.
} 
These terms lead to dimension five interactions violating baryon as well as lepton number. Such dimension five couplings are suppressed by just one power of the GUT scale and lead to too fast proton decay [23]. The authors of [4,5] proposed another discrete $\mathbb{Z}_{3}$ symmetry forbidding also the dimension five baryon number violating couplings. This symmetry was later dubbed baryon triality, $B_{3}$ [24, 25]. The charges are defined modulo hypercharge $(Y)$ transformation and listed in Table 1 .

Matter parity as well as baryon triality can be obtained from a spontaneously broken additional U(1) gauge symmetry [4,5]. Anomaly cancellation restricts the spectrum of a gauge theory leading to a finite number of possible discrete symmetries, which can be obtained in such a way. The authors of [6] looked through all such symmetries and identified proton hexality, $P_{6}$, as the only other phenomenologically interesting discrete symmetry. On SM fields $P_{6}$, as defined by the charge assignments in Table 1, acts as a $\mathbb{Z}_{6}$ symmetry which is the product of baryon triality and matter parity. Proton hexality had been discussed before in [26 29]. It forbids all dangerous dimension four and five baryon or lepton number violating couplings while phenomenologically desirable couplings are allowed. That is, only terms in the first line of (11) are allowed at the renormalisable level. At dimension five level, only interactions coming from a superpotential $L H_{u} L H_{u}$ (family indices are suppressed) are allowed. These respect baryon number and, moreover, provide Majorana mass terms for left-handed neutrinos after electroweak symmetry breaking. Dimension six interactions are suppressed by two powers of the GUT scale which results in a sufficiently stable proton for supersymmetric theories (with $M_{G U T} \sim 10^{16} \mathrm{GeV}$ ).

\begin{tabular}{|l||c|c|c|c|c|c|c|c|}
\hline & $Q$ & $\bar{U}$ & $\bar{D}$ & $L$ & $\bar{E}$ & $H_{u}$ & $H_{d}$ & $\bar{\nu}$ \\
\hline \hline $6 Y$ & 1 & -4 & 2 & -3 & 6 & 3 & -3 & 0 \\
\hline \hline $\mathbb{Z}_{2}^{\text {matter }}$ & 1 & 1 & 1 & 1 & 1 & 0 & 0 & 1 \\
$B_{3}$ & 0 & -1 & 1 & -1 & 2 & 1 & -1 & 0 \\
$P_{6}$ & 0 & 1 & -1 & -2 & 1 & -1 & 1 & 3 \\
\hline
\end{tabular}

Table 1: Hypercharge and discrete charges of the MSSM particles under hypercharge $Y$, matter parity $\mathbb{Z}_{2}^{\text {matter }}$, baryon triality $B_{3}$ and proton hexality $P_{6}$. One can show that $P_{6}=\mathbb{Z}_{2}^{\text {matter }} \times B_{3}$ up to a hypercharge shift. $\mathbb{Z}_{2}^{\text {matter }}, B_{3}$ and $P_{6}$ are defined just modulo 2,3 and 6 , respectively. A right-handed neutrino $\bar{\nu}$ has been included.

So far, our discussion did not include right-handed neutrinos. They can be included in a straightforward way (as shown in Table 1). Interactions including right-handed neutrinos do not introduce baryon number violating terms, since their $B_{3}$ charge is zero. All terms needed for the see-saw mechanism are allowed by $P_{6}[6]$.

\section{Proton Hexality and Unified Gauge Groups}

Since proton hexality forbids also dangerous dimension five couplings, it is desirable to embed $P_{6}$ into an underlying more fundamental theory. As a first example we look at 
grand unification. The embedding of $P_{6}$ into a GUT has been examined to some extent in [7. There, the authors added an extra anomaly free $U(1)$ to the unified gauge group and identified all possible discrete subgroups of that $\mathrm{U}(1)$. In this case, proton hexality does not work with any of the usual candidate unified gauge groups (Pati-Salam, SU(5), $\mathrm{SO}(10))$.

\subsection{Proton Hexality from Pati-Salam $\times \mathrm{U}(1)_{X}$}

An option which has been excluded in [7] is to break the unified gauge group times an extra $\mathrm{U}(1)_{X}$ simultaneously to the SM gauge group times $P_{6}$. For unified groups with rank larger than four $P_{6}$ can, in principle, be embedded diagonally into the extra $\mathrm{U}(1)_{X}$ times another $\mathrm{U}(1)$ originating from the GUT gauge group. Such a scheme works for Pati-Salam 3 with gauge group $\mathrm{SU}(4) \times \mathrm{SU}(2)_{L} \times \mathrm{SU}(2)_{R}$. The colour $\mathrm{SU}(3)$ is embedded into the upper three times three block of the hermitian $\mathrm{SU}(4)$ generator and hypercharge transformations are generated by a combination of an $\mathrm{SU}(4)$ and $\mathrm{SU}(2)_{R}$ transformation

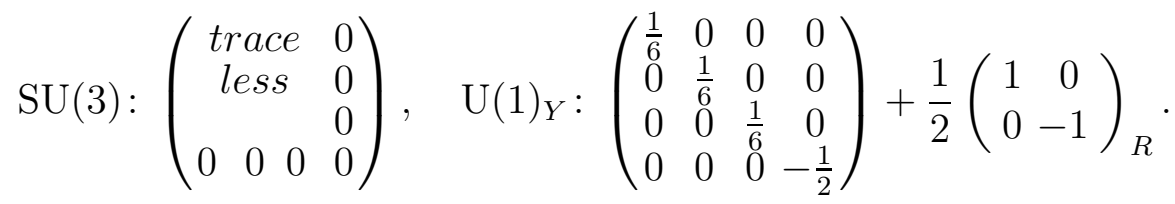

The left-handed quarks and leptons merge into a $(\mathbf{4}, \mathbf{2}, \mathbf{1})$ while the right-handed quarks and leptons form a $(\overline{\mathbf{4}}, \mathbf{1}, \mathbf{2})$ representation. The supersymmetric SM Higgs pair is combined into a $(\mathbf{1}, \mathbf{2}, \mathbf{2})$ representation. Now, we add an additional $\mathrm{U}(1)_{X}$ with generator $X$ and denote the corresponding charges by subscripts. With the assignments

$$
(4,2,1)_{1}, \quad(\overline{4}, 1,2)_{-1} \text { and }(\mathbf{1}, \mathbf{2}, 2)_{0}
$$

we reproduce the correct $P_{6}$ charges if we take $P_{6}$ to be the $\mathbb{Z}_{6}$ subgroup of the $\mathrm{U}(1)$ generated by

$$
P_{6}: \frac{1}{2}\left(\begin{array}{cccc}
1 & 0 & 0 & 0 \\
0 & 1 & 0 & 0 \\
0 & 0 & 1 & 0 \\
0 & 0 & 0 & -3
\end{array}\right)-\left(\begin{array}{cc}
1 & 0 \\
0 & -1
\end{array}\right)_{R}-\frac{1}{2} X .
$$

The spontaneous breaking to the SM gauge group times $P_{6}$ can be achieved by turning on a vacuum expectation value $(\mathrm{VEV})$ in the upper $\mathrm{SU}(2)_{R}$ component of a $(\mathbf{4}, \mathbf{1}, \mathbf{2})_{7}$ and the lower $\mathrm{SU}(2)_{R}$ component of a $(\overline{\mathbf{4}}, \mathbf{1}, \mathbf{2})_{-7}$. With respect to $\mathrm{SU}(4)$ the VEV points always into the fourth direction such that the colour $\mathrm{SU}(3)$ remains unbroken. Since these components carry $\mathrm{U}(1)_{P_{6}}$ charges \pm 6 (cf. eqn. (5) ), their VEVs leave $P_{6}$ unbroken. However, in a supersymmetric theory, we encounter mixed $\mathrm{SU}(2)_{L / R}^{2} \mathrm{U}(1)_{X}$ anomalies. For three families, these can be cancelled e.g. with the additional multiplets

$$
2 \times(\mathbf{1}, \mathbf{2}, \mathbf{1})_{-6}, \quad 2 \times(\mathbf{1}, \mathbf{1}, \mathbf{2})_{6} .
$$

\footnotetext{
${ }^{3}$ Other discrete symmetries suppressing proton decay within Pati-Salam and $\mathrm{SO}(10)$ have been identified in 30].
} 
These can have $\mathrm{SU}(3) \times \mathrm{SU}(2)_{L} \times \mathrm{U}(1)_{Y} \times P_{6}$ invariant mass terms and hence decouple at the breaking scale.

\subsection{Proton Hexality from $\mathrm{SO}(12)$}

As far as gauge coupling unification is concerned Pati-Salam is still characterised by three couplings and, in that sense, not quite a GUT yet. If we try to go one step further to $\mathrm{SO}(10)$, for instance, we are back at the problems discussed in [7]: the matter fields in eqn. (4) should merge into a $\mathbf{1 6}$ of $\mathrm{SO}(10)$. However, they cannot do that due to their opposite $\mathrm{U}(1)_{X}$ charges. The only way out, is to double the number of 16-dimensional representations. Then, obviously, only half of each representation gives rise to SM matter. However, for the remaining half representation not needed for matter a mechanism of multiplet splitting has to be invoked.

With that in mind we might as well consider gauge groups larger than $\mathrm{SO}(10)$ also accommodating the extra $\mathrm{U}(1)_{X}$. One canonical choice is $\mathrm{SO}(12)$. For this discussion it is more convenient to use Cartan-Weyl notation. There, a Lie algebra is given in the form

$$
\left[H_{i}, H_{j}\right]=0 \quad, \quad\left[H_{i}, E_{p}\right]=p_{i} E_{p}
$$

with $i=1, \ldots \operatorname{rank}(G)=6$ and the $p$ 's denote charge vectors, or roots, of the remaining generators. $\mathrm{SO}(12)$ has six Cartan generators $H_{1}, \ldots, H_{6}$ and the roots $p$ are given by

$$
(\underline{ \pm 1, \pm 1,0,0,0,0)} \text {. }
$$

Here, all 60 roots are generated by permuting underlined entries, resulting in the $60+$ $6=66$ dimensional adjoint of $\mathrm{SO}(12)$. Apart from the adjoint, the following $\mathrm{SO}(12)$ representations, specified by their weights, will be of interest for us

$$
\begin{aligned}
& \text { 32: ( } \pm \frac{1}{2}, \pm \frac{1}{2}, \pm \frac{1}{2}, \pm \frac{1}{2}, \pm \frac{1}{2}, \pm \frac{1}{2} \text { ) (even number of - signs), } \\
& \text { 32': ( } \pm \frac{1}{2}, \pm \frac{1}{2}, \pm \frac{1}{2}, \pm \frac{1}{2}, \pm \frac{1}{2}, \pm \frac{1}{2} \text { ) (odd number of - signs), } \\
& \text { 12: }( \pm 1,0,0,0,0,0) \text {. }
\end{aligned}
$$

The $\mathrm{SU}(4) \times \mathrm{SU}(2)_{L} \times \mathrm{SU}(2)_{R} \times \mathrm{U}(1)_{X}$ gauge group can be embedded into the adjoint of $\mathrm{SO}(12)$ as follows

$$
\begin{array}{ll}
\mathrm{SU}(4): & ( \pm 1, \pm 1,0,0,0,0), H_{1}, H_{2}, H_{3}, \\
\mathrm{SU}(2)_{R}: & \mathrm{SU}(2)_{L}: \pm(0,0,0,1,-1,0), H_{4}-H_{5}, \\
\pm(0,0,0,1,1,0), H_{4}+H_{5}, & \mathrm{U}(1)_{X}:-2 H_{6} .
\end{array}
$$

Quarks and leptons become part of $\mathbf{3 2}$ and $\mathbf{3 2} \mathbf{2}^{\prime}$ representations,

$$
\begin{array}{ll}
(\mathbf{4}, \mathbf{2}, \mathbf{1})_{1}: & (\underbrace{ \pm \frac{1}{2}, \pm \frac{1}{2}, \pm \frac{1}{2}}_{\text {even } \#-}, \underbrace{ \pm \frac{1}{2}, \pm \frac{1}{2}}_{\text {odd } \#-},-\frac{1}{2}) \subset 32 \\
(\overline{4}, \mathbf{1}, \mathbf{2})_{-1}: & (\underbrace{ \pm \frac{1}{2}, \pm \frac{1}{2}, \pm \frac{1}{2}}_{\text {odd } \#-}, \underbrace{ \pm \frac{1}{2}, \pm \frac{1}{2}}_{\text {even } \#-}, \frac{1}{2}) \subset \mathbf{3 2},
\end{array}
$$


for each generation. Last, the electroweak Higgs sits inside a 12-dimensional representation

$$
(\mathbf{1}, \mathbf{2}, \mathbf{2})_{0}: \quad(0,0,0, \pm 1,0,0) \subset \mathbf{1 2} .
$$

Clearly, these multiplets have a fair amount of additional fields which need to decouple via multiplet splitting. The situation becomes much more dramatic for the remaining fields: $(\mathbf{4}, \mathbf{1}, \mathbf{2})_{7},(\overline{\mathbf{4}}, \mathbf{1}, \mathbf{2})_{-7}$ and the fields in eqn. (6). These are embedded in $\mathrm{SO}(12)$ representations containing weights of the form $( \pm 7 / 2, \ldots, \pm 7 / 2)$ and $(0, \ldots, 0, \pm 3)$, respectively. The big charges under one of the $\mathrm{SO}(12)$ Cartan generators can be accommodated only in rather high-dimensional representations 4 Even with a working mechanism for multiplet splitting one would rather not add such representations to an underlying fundamental theory 5 Therefore, we should also have the option of adding incomplete or split multiplets. In the next section we will demonstrate that both mechanisms can be naturally obtained within string theory.

\section{Proton Hexality and Local Grand Unification}

The picture of local grand unification has drawn considerable attention in the recent past in particular in the context of heterotic model building (for recent reviews, see [32, 33]). Before discussing orbifold compactifications of the heterotic string, let us demonstrate how the previous problems are solved in a bottom-up approach with two extra dimensions. 6 . For simplicity, we construct the previously described Pati-Salam times $U(1)_{X}$ model in four dimensions. When discussing actual string models, later on, we will be interested in four-dimensional gauge symmetry of the form: SM gauge group times $P_{6}$ (times hidden sector gauge group). Since the step from Pati-Salam times $\mathrm{U}(1)_{X}$ to the $\mathrm{SM}$ times $P_{6}$ is comparatively simple there is no conceptual difference.

Two extra dimensions are compactified on a torus obtained by modding the complex plane with a quadratic lattice spanned by the vectors $e_{1}$ and $e_{2}$. Further we mod out a $\mathbb{Z}_{4}$ symmetry generated by $\pi / 2$ rotations in the plane. The fixed point structure is depicted in Figure 1. As a six-dimensional theory we take an $\mathrm{SO}(12)$ gauge theory. The $\pi / 2$ rotation is embedded into $\mathrm{SO}(12)$ by the adjoint action of $e^{2 \pi \mathrm{i} H_{j} V_{j}}$ and lattice shifts by $e_{1}$ or $e_{2}$ by $e^{2 \pi \mathrm{i} H_{j} W_{j}}$. (Shifts by $e_{1}$ and $e_{2}$ have to be embedded identically since $e_{1}$ is mapped onto $e_{2}$ by the $\pi / 2$ rotation.) We choose

$$
V=\left(\frac{1}{2}, \frac{1}{2}, \frac{1}{2}, 0,0,0\right), W=\left(0,0,0,0,0, \frac{1}{2}\right) .
$$

\footnotetext{
${ }^{4}$ The corresponding highest weights are $\left(\frac{7}{2}, \frac{7}{2}, \frac{7}{2}, \frac{7}{2}, \frac{7}{2}, \frac{7}{2}\right)$ and $(3,0,0,0,0,0)$ with Dynkin labels $[0,0,0,0,0,7]$ and $[3,0,0,0,0,0]$. The dimensions can be computed using the Weyl formula (see e.g. [31]) and are $2,617,472$ and 352 .

${ }^{5}$ Note that in the case of matter parity as a subgroup of $\mathrm{SO}(10)$ we would need a 126-dimensional representation to get the desired symmetry breakdown.

${ }^{6}$ In the spirit of [14] this may correspond to a compactification of the heterotic string on $T^{6} / \mathbb{Z}_{M} \times \mathbb{Z}_{N}$, where our $T^{2}$ appears as a fixed torus under the $\mathbb{Z}_{N}$ factor. For a list of possible $\mathbb{Z}_{M} \times \mathbb{Z}_{N}$ orbifolds see e.g. 34].
} 


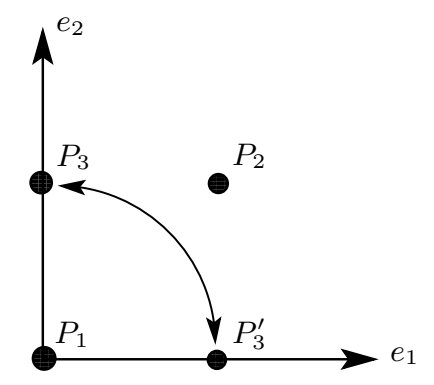

Figure 1: Fixed point structure of $T^{2} / \mathbb{Z}_{4}$ orbifold. The origin $P_{1}$ is fixed under any rotation. $P_{2}$ is fixed under a $\pi / 2$ rotation followed by a shift by $e_{1} . P_{3}$ and $P_{3}^{\prime}$ are fixed under a $\pi$ rotation and shifts by $e_{2}$ and $e_{1}$, respectively. $P_{3}$ and $P_{3}^{\prime}$ are related by a $\pi / 2$ rotation and thus identical on the orbifold.

The unbroken gauge group in four dimensions is the subgroup of $\mathrm{SO}(12)$ which is invariant under the orbifold action and lattice shifts, i.e. $p \cdot V=0 \bmod 1$ and $p \cdot W=0 \bmod 1, p$ being a root of $\mathrm{SO}(12)$. This yields Pati-Salam times $\mathrm{U}(1)_{X}$ embedded into $\mathrm{SO}(12)$ as discussed in the previous section eqn. (10). To avoid enormous representations, the $(\mathbf{4}, \mathbf{1}, \mathbf{2})_{7}$ and $(\overline{4}, 1,2)_{-7}$ and fields in (6) should be localised to points where $\mathrm{U}(1)_{X}$ factorises and a big charge does not need a large representation. The gauge group geography showing the local projections of $\mathrm{SO}(12)$ at the fixed points is depicted in Figure 2. At the fixed point(s)

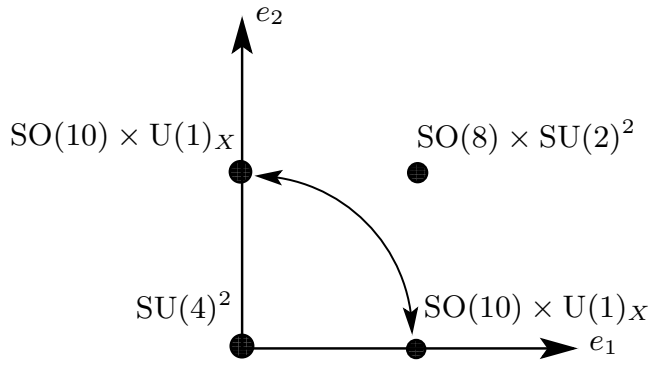

Figure 2: Gauge group geography of $T^{2} / \mathbb{Z}_{4}$ orbifold. The gauge group in the bulk is $\mathrm{SO}(12)$.

with $\mathrm{SO}(10) \times \mathrm{U}(1)_{X}$ gauge group we localise a $2 \times \mathbf{1 0}_{6}, 2 \times \mathbf{1 0}_{-6}, \mathbf{1 6}_{7}, \overline{\mathbf{1 6}}_{-7}$. From an $\mathrm{SO}(12)$ perspective these are split multiplets of dramatically lower dimension than complete multiplets containing fields of the same $\mathrm{U}(1)_{X}$ charges. We demonstrate the mechanism of multiplet splitting by getting SM matter and Higgses from complete $\mathrm{SO}(12)$ multiplets in the bulk. For each family of quarks and leptons we add a 32 and a $\mathbf{3 2}$-plet and for the electroweak Higgs a 12-plet to the bulk. The action of orbifold and lattice shifts is again given by $e^{2 \pi \mathrm{i} H_{j} V_{j}}$ and $e^{2 \pi \mathrm{i} H_{j} W_{j}}$ in the corresponding representation. In addition it is accompanied by phase factors called orbifold parities. (For a discussion within the context of heterotic orbifolds, see e.g. 35].) In our bottom-up approach we pick the phases by hand. With an appropriate choice, it is easy to project the multiplets exactly to the desired $\mathrm{SU}(4) \times \mathrm{SU}(2)_{L} \times \mathrm{SU}(2)_{R} \times \mathrm{U}(1)_{X}$ multiplets in the four-dimensional effective theory. 
There are still six-dimensional bulk anomalies to worry about. The relevant formulæ can be found e.g. in [36]. To cancel the bulk anomalies, we have to add 16 12-plets to the bulk theory if all three families originate from bulk multiplets. We will not get further into the details of our illustrative example. After all, this theory is not UV complete and what we are really after is a fully-fledged string model.

\section{Embedding Proton Hexality in Heterotic Orbifolds}

\subsection{Proton Hexality from local GUTs}

In this section, we are interested in how to obtain string compactifications furnished with gauged proton hexality. We follow the strategy depicted in the previous section, i.e. we search for MSSM-like constructions in which $P_{6}$ arises as a subgroup of $\mathrm{SU}(4) \times \mathrm{SU}(2)_{L} \times$ $\mathrm{SU}(2)_{R} \times \mathrm{U}(1)_{X} \subset \mathrm{SO}(12)$ and the matter generations reside in (split) representations 32 and $3 \mathbf{2}^{\prime}$ of the six-dimensional SO(12) GUT. With this purpose, we consider the $T^{6} / \mathbb{Z}_{4} \times \mathbb{Z}_{4}$ orbifold with torus lattice $\mathrm{SO}(5)^{3}$. The $\mathbb{Z}_{4} \times \mathbb{Z}_{4}$ action is generated by the twists

$$
v_{1}=\left(\frac{1}{4}, 0,-\frac{1}{4}\right) \quad \text { and } \quad v_{2}=\left(0, \frac{1}{4},-\frac{1}{4}\right) .
$$

This orbifold allows for one Wilson line of order 2 per $\mathrm{SO}(5)$ torus, hence three independent Wilson lines. Note that, since the twist $v_{1}\left(v_{2}\right)$ leaves the second (first) $\mathrm{SO}(5)$ torus invariant, it defines a $T^{4} / \mathbb{Z}_{4}$ orbifold.

Along the lines of the heterotic Mini-Landscape [10,11, we apply a search strategy based on local GUTs to find a fertile region of heterotic orbifolds endowed with proton hexality. The search strategy reads as follows. To start with, we consider the first $\mathbb{Z}_{4}$. From all its possible gauge embeddings $V_{1}$ [37, 38], we choose the shift vector (denoted by $(\mathrm{IVg})$ in [38])

$$
V_{1}=\left(\frac{3}{4}, \frac{1}{4}, 0^{6}\right)\left(0^{8}\right),
$$

which results in a $T^{4} / \mathbb{Z}_{4}$ model with $\mathrm{SO}(12)$ gauge group and twisted matter states transforming as $\mathbf{3 2}$ or $\mathbf{3 2}$-plets. We look for all admissible gauge embeddings $V_{2}$ associated to the second $\mathbb{Z}_{4}$ action, resulting in 164 possibilities and select only those that break $\mathrm{SO}(12)$ to Pati-Salam,

$$
\mathrm{SO}(12) \stackrel{V_{2}}{\longrightarrow} \mathrm{SU}(4) \times \mathrm{SU}(2)_{L} \times \mathrm{SU}(2)_{R} \times \mathrm{U}(1)_{X} .
$$

This reduces the number of promising shifts $V_{2}$ dramatically from 164 to 30 . In addition, we want SM matter representations arising from 32 and $32^{\prime}$-plets, i.e. quarks and leptons shall stem from the twisted sectors of the underlying $T^{4} / \mathbb{Z}_{4}$ orbifold. Hence, we choose only those 12 shifts $V_{2}$ out of the 30 where the $(i, 0)$-twisted sector $(i=1,2,3)$ of the full $T^{6} / \mathbb{Z}_{4} \times \mathbb{Z}_{4}$ contains some Pati-Salam matter representations.

The final step is to select models with up to two Wilson lines that yield the gauge group and matter spectrum of the MSSM plus vector-like exotics. With the help of the methods developed in [12], we find more than 850 heterotic orbifolds with the desired properties. In many of these models, there is a $\mathrm{U}(1)$ that by construction, leads to (at least some) quarks 
and leptons with the correct $P_{6}$ charges and, therefore, suppression of proton decay occurs automatically. However, the models we were able to construct suffer from exotics that are not vector-like w.r.t. $P_{6}$. In what follows we present an example of these constructions.

An example The model is defined by the shift $V_{1}$ of eqn. (15), the second shift

$$
V_{2}=\left(-\frac{3}{4}, \frac{1}{2},-\frac{1}{2}, 0^{3}, \frac{1}{4}, \frac{1}{2}\right)\left(-1,0^{3}, \frac{1}{4}^{4}\right),
$$

and one Wilson line $W_{2}$ associated to the $e_{2}$ direction

$$
W_{2}=\left(-\frac{3}{4}, \frac{9}{4},-\frac{5}{4},-\frac{3}{4},-\frac{1}{4}, \frac{5}{4}, \frac{1}{4},-\frac{3}{4}\right)\left(\frac{1}{2}^{4}, 0^{3}, 2\right) .
$$

Schematically, the gauge symmetry is broken as expected:

$$
\mathrm{E}_{8} \stackrel{V_{1}}{\longrightarrow} \mathrm{SO}(12) \stackrel{V_{2}}{\longrightarrow} \mathrm{PS} \times \mathrm{U}(1)_{X} \stackrel{W_{2}}{\longrightarrow} \mathrm{SM}
$$

resulting in the four-dimensional gauge group

$$
\mathrm{SU}(3) \times \mathrm{SU}(2) \times \mathrm{U}(1)_{Y} \times \mathrm{U}(1)^{4} \times[\mathrm{SU}(4) \times \mathrm{SO}(10)] .
$$

One of the U(1)'s, orthogonal to $\mathrm{U}(1)_{Y}$, appears anomalous.

Using the $\mathrm{U}(1)_{X}$ direction orthogonal to Pati-Salam, but inside $\mathrm{SO}(12)$ we can define a (non-anomalous) generator along the lines of eqn. (51). Explicitly, it reads

$$
t_{P_{6}}=(0,0,2,-2,2,-2,2,-2)\left(0^{8}\right) .
$$

This is our prime choice for a $\mathrm{U}(1)$ proton hexality. In fact, the spectrum contains three generations of quarks and leptons with the right charge assignments and four SM singlets with $\mathrm{U}(1)_{P_{6}}$ charge 6 that can be used to break $\mathrm{U}(1)_{P_{6}}$ to its discrete subgroup $P_{6}$,

$$
3(\mathbf{3}, \mathbf{2})_{\frac{1}{6}, 0}+3(\overline{\mathbf{3}}, \mathbf{1})_{-\frac{2}{3}, 1}+3(\overline{\mathbf{3}}, \mathbf{1})_{\frac{1}{3},-1}+3(\mathbf{1}, \mathbf{2})_{-\frac{1}{2}, 4}+3(\mathbf{1}, \mathbf{1})_{1,1}+3(\mathbf{1}, \mathbf{1})_{0,-3}+4(\mathbf{1}, \mathbf{1})_{0,6} .
$$

See Table 2 for the full massless matter spectrum. The spectrum contains in addition exotics that are vector-like with respect to the SM. Unfortunately, these exotics turn out to be chiral with respect to $P_{6}$. Hence, they can only decouple once $P_{6}$ is broken. One would have to make sure that this breakdown is compatible with the desired proton stability. These questions will be studied in a future publication.

\subsection{Proton Hexality as Accidental Symmetry}

We have focused so far on exact gauge symmetries of orbifold compactifications and how they can be broken to give rise to $P_{6}$. Alternatively, one can consider the large set of approximate $\mathrm{U}(1)$ symmetries that arise naturally in orbifold models and lead to solutions of certain issues, such as the strong CP-problem [39, 40]. 
The general procedure to address this question is as follows. Considering the effective superpotential $\mathcal{W}$ truncated at a given order, one has to identify the $\mathrm{U}(1)$ symmetries (other than the gauged ones) under which $\mathcal{W}$ is invariant. Then one must require that a linear combination $\mathrm{U}(1)_{P_{6}}$ of these $\mathrm{U}(1)$ s provide the correct charge assignments for all the SM-fields (see Table 1) and that there be at least one SM-singlet $\chi$ with charges \pm 6 whose VEV breaks $\mathrm{U}(1)_{P_{6}}$ down to $P_{6}$. One must further enforce that the spectrum be three generations plus vector-like matter also w.r.t. $\mathrm{U}(1)_{P_{6}}$.

We have performed a search of such symmetries among the models of the $\mathbb{Z}_{6}$-II MiniLandscape with two and three Wilson lines and found no example displaying three generations with the standard $P_{6}$ charges. If one relaxes this condition and requires correct $P_{6}$ only for the first two generations, there are some examples. However, they do not satisfy all the requirements listed before. In the following we present a model of this type. It is not clear whether better models are possible in this scenario. In fact the strategy employed in the construction of the Mini-Landscape [10,11] aiming preferentially at complete multiplets could be incompatible with the incorporation of $P_{6}$ in a satisfactory way.

An example Let us consider the $\mathbb{Z}_{6}-$ II orbifold model described by the shift embedding

$$
V^{\mathrm{E}_{6}, 1}=\left(\frac{1}{6},-\frac{1}{3},-\frac{1}{2}, 0,0,0,0,0\right)(0,0,0,0,0,0,0,0)
$$

and the Wilson lines

$$
\begin{aligned}
& W_{3}=\left(-\frac{5}{6},-\frac{7}{6}, \frac{1}{2}, \frac{1}{2}, \frac{1}{2},-\frac{1}{2},-\frac{1}{2},-\frac{1}{2}\right)\left(0,0, \frac{1}{3}, \frac{1}{3}, \frac{1}{3}, 0,1, \frac{2}{3}\right), \\
& W_{2}=\left(1, \frac{1}{2}, 0, \frac{1}{2}, \frac{1}{2},-\frac{1}{2},-1,0\right)\left(-\frac{1}{4}, \frac{3}{4}, \frac{1}{4}, \frac{1}{4}, \frac{3}{4},-\frac{3}{4},-\frac{3}{4}, \frac{3}{4}\right), \\
& W_{2}^{\prime}=\left(\frac{3}{4}, \frac{3}{4},-\frac{1}{4},-\frac{1}{4},-\frac{1}{4}, \frac{3}{4}, \frac{1}{4}, \frac{1}{4}\right)\left(-\frac{1}{4},-\frac{1}{4},-\frac{1}{4},-\frac{1}{4},-\frac{1}{4}, \frac{1}{4}, \frac{1}{4}, \frac{3}{4}\right) .
\end{aligned}
$$

The four-dimensional gauge group is $\mathrm{SU}(3) \times \mathrm{SU}(2)_{L} \times \mathrm{U}(1)_{Y} \times\left[\mathrm{SU}(6) \times \mathrm{U}(1)^{7}\right]$ with $\mathrm{U}(1)_{Y} \subset \mathrm{SU}(5)$, and the massless spectrum includes three SM generations plus vector-like exotics with respect to the SM gauge group.

At order four in the superpotential, apart from the gauge $\mathrm{U}(1) \mathrm{s}$, there appear 82 accidental U(1)s. A linear combination of all the available U(1)s renders the following properties (cf. Table 3):

a) there is a set of SM singlets $\chi$ that break $\mathrm{U}(1)_{P_{6}}$ to $P_{6}$;

b) two SM families (including right-handed neutrinos) have proper $P_{6}$ charges;

c) a pair of Higgs fields $h_{u}, h_{d}$ have proper $P_{6}$ charges; and

d) apart from a third generation with unwanted $P_{6}$ charges, all other exotic states are vector-like.

This model has the potential to forbid proton decay. However, there are still some questions to be analysed. First, in this specific model, operators of order five in the superpotential break explicitly $\mathrm{U}(1)_{P_{6}}$. Secondly, a large mass for the top-quark is forbidden. Finally, $\mathrm{U}(1)_{P_{6}}$ exhibits anomalies such as $\operatorname{tr} Q_{P_{6}}^{3}, \operatorname{tr} Q_{P_{6}}^{2} Q_{Y}, \operatorname{tr} Q_{Y}^{2} Q_{P_{6}} \neq 0$, that would have to be cancelled. A search for a more realistic model is under way. 


\section{Conclusions}

Proton decay is a crucial question in grand unified theories. The proton should decay, but not too fast. In supersymmetric theories we have to forbid dim- 4 and -5 operators. Usual matter parity is not enough as it still allows dangerous dim-5 operators. In this respect proton hexality is perfect. It forbids all the couplings that we do not want and allows those we need.

For a long time, its incompatibility with grand unification was thought to be a problem. The concept of "local grand unification" discussed in string theories (and theories of extra dimensions), however, changes the picture. Split multiplets, solving already the problem of doublet-triplet splitting and the question of breakdown of the grand unified gauge group, come to rescue and make hexality potentially compatible with models that have been constructed in the framework of (heterotic) string theory.

Still the search for a fully realistic model requires more work and perhaps a dedicated search strategy. Models as discussed e.g. in the Mini-Landscape [10,11] are not so well suited here by construction, as they were based on the desire to have unified multiplets for the first two families. Hexality would require a different approach and it would be desirable to set up a general geometric picture that naturally incorporates $P_{6}$.

So far we have learned some lessons from string theory. Hexality can come from various sources. It could be

- a subgroup of a non-anomalous symmetry,

- a subgroup of an anomalous symmetry,

- an accidental symmetry.

In fact, $P_{6}$ could just be an approximate symmetry that is valid at the level of lowerdimensional operators or valid only for part of the spectrum (like the first and second family).

It is worthwhile to explore these questions further, both from the bottom-up and topdown approaches. The present model building attempts just scratch the surface of the landscape. More dedicated model building is needed to construct fully realistic models. We have here presented some toy models that illustrate the potential marriage of hexality with local grand unification. We are confident to report about the construction of more realistic models in the near future.

\section{Acknowledgments}

P.V. would like to thank LMU Excellent for support. S.R-S. is grateful to P. Frampton, K. Wang and T. Yanagida for useful discussions at IPMU. This research was supported by the DFG cluster of excellence Origin and Structure of the Universe, the SFB-Tansregio TR33 "The Dark Universe" (Deutsche Froschungsgemeinschaft) and the European Union 7th network program "Unification in the LHC era" (PITN-GA-2009-237920). 


\section{A Spectra of Toy Models}

\begin{tabular}{|clc|}
\hline$\#$ & Irrep & \\
\hline 4 & $(\mathbf{3}, \mathbf{2}, \mathbf{1}, \mathbf{1})_{(1 / 6,0)}$ & $q$ \\
1 & $(\mathbf{3}, \mathbf{2}, \mathbf{1}, \mathbf{1})_{(1 / 6,1)}$ & $q^{\prime}$ \\
2 & $(\overline{\mathbf{3}}, \mathbf{2}, \mathbf{1}, \mathbf{1})_{(-1 / 6,2)}$ & $\bar{q}^{\prime}$ \\
\hline 3 & $(\overline{\mathbf{3}}, \mathbf{1}, \mathbf{1}, \mathbf{1})_{(-2 / 3,1)}$ & $\bar{u}$ \\
\hline 3 & $(\overline{\mathbf{3}}, \mathbf{1}, \mathbf{1}, \mathbf{1})_{(1 / 3,-1)}$ & $\bar{d}$ \\
3 & $(\overline{\mathbf{3}}, \mathbf{1}, \mathbf{1}, \mathbf{1})_{(1 / 3,1)}$ & $\bar{d}^{\prime}$ \\
3 & $(\overline{\mathbf{3}}, \mathbf{1}, \mathbf{1}, \mathbf{1})_{(1 / 3,-2)}$ & $\bar{d}^{\prime}$ \\
1 & $(\overline{\mathbf{3}}, \mathbf{1}, \mathbf{1}, \mathbf{1})_{(1 / 3,2)}$ & $\bar{d}^{\prime}$ \\
\hline
\end{tabular}

\begin{tabular}{|clc|}
\hline$\#$ & Irrep & \\
\hline 1 & $(\overline{\mathbf{3}}, \mathbf{1}, \mathbf{1}, \mathbf{1})_{(1 / 3,-4)}$ & $\bar{d}^{\prime}$ \\
2 & $(\mathbf{3}, \mathbf{1}, \mathbf{1}, \mathbf{1})_{(-1 / 3,2)}$ & $d^{\prime}$ \\
3 & $(\mathbf{3}, \mathbf{1}, \mathbf{1}, \mathbf{1})_{(-1 / 3,-2)}$ & $d^{\prime}$ \\
3 & $(\mathbf{3}, \mathbf{1}, \mathbf{1}, \mathbf{1})_{(-1 / 3,-1)}$ & $d^{\prime}$ \\
\hline 4 & $(\mathbf{1}, \mathbf{2}, \mathbf{1}, \mathbf{1})_{(-1 / 2,4)}$ & $\ell$ \\
7 & $(\mathbf{1}, \mathbf{2}, \mathbf{1}, \mathbf{1})_{(-1 / 2,-2)}$ & $\ell$ \\
1 & $(\mathbf{1}, \mathbf{2}, \mathbf{1}, \mathbf{1})_{(-1 / 2,2)}$ & $\ell^{\prime}$ \\
1 & $(\mathbf{1}, \mathbf{2}, \mathbf{1}, \mathbf{1})_{(-1 / 2,-1)}$ & $\ell^{\prime}$ \\
\hline
\end{tabular}

\begin{tabular}{|rlc|}
\hline$\#$ & Irrep & \\
\hline 8 & $(\mathbf{1}, \mathbf{2}, \mathbf{1}, \mathbf{1})_{(1 / 2,2)}$ & $\bar{\ell}^{\prime}$ \\
1 & $(\mathbf{1}, \mathbf{2}, \mathbf{1}, \mathbf{1})_{(1 / 2,4)}$ & $\bar{\ell}^{\prime}$ \\
1 & $(\mathbf{1}, \mathbf{2}, \mathbf{1}, \mathbf{1})_{(1 / 2,-2)}$ & $\bar{\ell}^{\prime}$ \\
\hline 9 & $(\mathbf{1}, \mathbf{2}, \mathbf{1}, \mathbf{1})_{(1 / 2,-1)}$ & $h_{u}$ \\
9 & $(\mathbf{1}, \mathbf{2}, \mathbf{1}, \mathbf{1})_{(-1 / 2,1)}$ & $h_{d}$ \\
\hline 3 & $(\mathbf{1}, \mathbf{1}, \mathbf{1}, \mathbf{1})_{(1,1)}$ & $\bar{e}$ \\
\hline 18 & $(\mathbf{1}, \mathbf{1}, \mathbf{1}, \mathbf{1})_{(0,-3)}$ & $\bar{\nu}$ \\
15 & $(\mathbf{1}, \mathbf{1}, \mathbf{1}, \mathbf{1})_{(0,3)}$ & \\
\hline
\end{tabular}

\begin{tabular}{|rlc|}
\hline 3 & $(\mathbf{1}, \mathbf{1}, \mathbf{1}, \mathbf{1})_{(0,6)}$ & $\chi$ \\
1 & $(\mathbf{1}, \mathbf{1}, \mathbf{1}, \mathbf{1})_{(0,-6)}$ & $\chi$ \\
18 & $(\mathbf{1}, \mathbf{1}, \mathbf{1}, \mathbf{1})_{(0,0)}$ & $s^{0}$ \\
\hline 8 & $(\mathbf{1}, \mathbf{2}, \overline{\mathbf{4}}, \mathbf{1})_{(0,-1 / 2)}$ & $m$ \\
2 & $(\mathbf{1}, \mathbf{2}, \mathbf{4}, \mathbf{1})_{(0,-5 / 2)}$ & $m^{\prime}$ \\
\hline 6 & $(\mathbf{1}, \mathbf{1}, \mathbf{4}, \mathbf{1})_{(-1 / 2,3 / 2)}$ & $s^{-}$ \\
2 & $(\mathbf{1}, \mathbf{1}, \mathbf{4}, \mathbf{1})_{(-1 / 2,-3 / 2)}$ & $s^{-}$ \\
\hline
\end{tabular}

\begin{tabular}{|llc|}
\hline 4 & $(\mathbf{1}, \mathbf{1}, \overline{\mathbf{4}}, \mathbf{1})_{(-1 / 2,-5 / 2)}$ & $s^{-}$ \\
2 & $(\mathbf{1}, \mathbf{1}, \mathbf{4}, \mathbf{1})_{(1 / 2,5 / 2)}$ & $s^{+}$ \\
4 & $(\mathbf{1}, \mathbf{1}, \mathbf{4}, \mathbf{1})_{(1 / 2,-7 / 2)}$ & $s^{+}$ \\
4 & $(\mathbf{1}, \mathbf{1}, \mathbf{4}, \mathbf{1})_{(1 / 2,-1 / 2)}$ & $s^{+}$ \\
2 & $(\mathbf{1}, \mathbf{1}, \overline{\mathbf{4}}, \mathbf{1})_{(1 / 2,1 / 2)}$ & $s^{+}$ \\
\hline 5 & $(\mathbf{1}, \mathbf{1}, \mathbf{6}, \mathbf{1})_{(0,2)}$ & $x$ \\
6 & $(\mathbf{1}, \mathbf{1}, \mathbf{6}, \mathbf{1})_{(0,1)}$ & \\
\hline
\end{tabular}

\begin{tabular}{|lll|}
\hline 1 & $(\mathbf{1}, \mathbf{1}, \mathbf{1}, \mathbf{1 0})_{(0,2)}$ & $y$ \\
2 & $(\mathbf{1}, \mathbf{1}, \mathbf{1}, \mathbf{1 0})_{(0,-2)}$ & \\
2 & $(\mathbf{1}, \mathbf{1}, \mathbf{1}, \mathbf{1 0})_{(0,1)}$ & \\
\hline 2 & $(\mathbf{1}, \mathbf{1}, \mathbf{1}, \mathbf{1})_{(0,2)}$ & $\tilde{s}$ \\
7 & $(\mathbf{1}, \mathbf{1}, \mathbf{1}, \mathbf{1})_{(0,-2)}$ & \\
3 & $(\mathbf{1}, \mathbf{1}, \mathbf{1}, \mathbf{1})_{(0,1)}$ & \\
9 & $(\mathbf{1}, \mathbf{1}, \mathbf{1}, \mathbf{1})_{(0,-1)}$ & \\
\hline
\end{tabular}

Table 2: Massless spectrum of a model with gauged $P_{6}$. Quantum numbers w.r.t. $\left[\mathrm{SU}(3)_{C} \times\right.$ $\left.\mathrm{SU}(2)_{\mathrm{L}}\right] \times[\mathrm{SU}(4) \times \mathrm{SO}(10)]$ (bold) and $\mathrm{U}(1)_{Y} \times \mathrm{U}(1)_{P_{6}}$ (subscripts) are given.

\begin{tabular}{|c|c|c|c|c|c|}
\hline$\#$ & Irrep & & $\#$ & Anti-irrep & \\
\hline 2 & $(\mathbf{3}, \mathbf{2} ; \mathbf{1})_{(1 / 6,0)}$ & $q_{1,2}$ & & & \\
\hline 1 & $(\mathbf{3}, \mathbf{2} ; \mathbf{1})_{(1 / 6,25 / 2)}$ & $q_{3}$ & & & \\
\hline 1 & $(\mathbf{3}, \mathbf{2} ; \mathbf{1})_{(1 / 6,7 / 2)}$ & $q^{\prime}$ & 1 & $(\overline{\mathbf{3}}, \mathbf{2} ; \mathbf{1})_{(-1 / 6,-7 / 2)}$ & $\bar{q}^{\prime}$ \\
\hline 2 & $(\mathbf{1}, \mathbf{2} ; \mathbf{1})_{(-1 / 2,-2)}$ & $\ell_{1,2}$ & & & \\
\hline 1 & $(\mathbf{1}, \mathbf{2} ; \mathbf{1})_{(-1 / 2,0)}$ & $\ell_{3}$ & & & \\
\hline 1 & $(\mathbf{1}, \mathbf{2} ; \mathbf{1})_{(-1 / 2,1)}$ & $h_{d}$ & 1 & $(\mathbf{1}, \mathbf{2} ; \mathbf{1})_{(1 / 2,-1)}$ & $h_{u}$ \\
\hline 2 & $(\mathbf{1}, \mathbf{2} ; \mathbf{1})_{(-1 / 2,2)}$ & $\ell^{\prime}$ & 2 & $(\mathbf{1}, \mathbf{2} ; \mathbf{1})_{(1 / 2,-2)}$ & $\bar{\ell}^{\prime}$ \\
\hline 1 & $(\mathbf{1}, \mathbf{2} ; \mathbf{1})(-1 / 2,-1 / 2)$ & & 1 & $(\mathbf{1}, \mathbf{2} ; \mathbf{1})_{(1 / 2,1 / 2)}$ & \\
\hline 1 & $(\mathbf{1}, \mathbf{2} ; \mathbf{1})_{(-1 / 2,3)}$ & & 1 & $(\mathbf{1}, \mathbf{2} ; \mathbf{1})_{(1 / 2,3)}$ & \\
\hline 2 & $(\overline{\mathbf{3}}, \mathbf{1} ; \mathbf{1})_{(1 / 3,-1)}$ & $d_{1,2}$ & & & \\
\hline 1 & $(\overline{\mathbf{3}}, \mathbf{1} ; \mathbf{1})_{(1 / 3,0)}$ & $\bar{d}_{3}$ & & & \\
\hline 3 & $(\overline{\mathbf{3}}, \mathbf{1} ; \mathbf{1})_{(1 / 3,2)}$ & $\bar{d}^{\prime}$ & 3 & $(\mathbf{3}, \mathbf{1} ; \mathbf{1})_{(-1 / 3,-2)}$ & $d^{\prime}$ \\
\hline 1 & $(\overline{\mathbf{3}}, \mathbf{1} ; \mathbf{1})_{(1 / 3,0)}$ & & 1 & $(\mathbf{3}, \mathbf{1} ; \mathbf{1})_{(-1 / 3,6)}$ & \\
\hline 1 & $(\overline{\mathbf{3}}, \mathbf{1} ; \mathbf{1})_{(1 / 3,1 / 2)}$ & & 1 & $(\mathbf{3}, \mathbf{1} ; \mathbf{1})_{(-1 / 3,-1 / 2)}$ & \\
\hline 2 & $(\overline{\mathbf{3}}, \mathbf{1} ; \mathbf{1})_{(-2 / 3,1)}$ & $\bar{u}_{1,2}$ & & & \\
\hline 1 & $(\overline{\mathbf{3}}, \mathbf{1} ; \mathbf{1})_{(-2 / 3,9 / 2)}$ & $\bar{u}_{3}$ & & & \\
\hline 1 & $(\overline{\mathbf{3}}, \mathbf{1} ; \mathbf{1})_{(-2 / 3,-7 / 9)}$ & $\bar{u}^{\prime}$ & 1 & $(\mathbf{3}, \mathbf{1} ; \mathbf{1})_{(2 / 3,7 / 9)}$ & $u^{\prime}$ \\
\hline 3 & $(\mathbf{1}, \mathbf{1} ; \mathbf{1})_{(1,1)}$ & $\bar{e}_{1,2,3}$ & & & \\
\hline 1 & $(\mathbf{1}, \mathbf{1} ; \mathbf{1})_{(1,0)}$ & $\bar{e}^{\prime}$ & 1 & $(\mathbf{1}, \mathbf{1} ; \mathbf{1})_{(-1,12)}$ & $e^{\prime}$ \\
\hline 1 & $(\mathbf{3}, \mathbf{1} ; \mathbf{1})_{(1 / 6,25 / 31)}$ & $\bar{v}$ & 1 & $(\overline{\mathbf{3}}, \mathbf{1} ; \mathbf{1})_{(-1 / 6,-25 / 31)}$ & $\bar{v}$ \\
\hline 1 & $(\mathbf{1}, \mathbf{1} ; \overline{\mathbf{6}})_{(1 / 2,40 / 27)}$ & $w^{+}$ & 1 & $(\mathbf{1}, \mathbf{1} ; \mathbf{6})_{(-1 / 2,-40 / 27)}$ & $w^{-}$ \\
\hline 3 & $(\mathbf{1}, \mathbf{1} ; \mathbf{1})_{(1 / 2,2 / 27)}$ & $s^{+}$ & 3 & $(\mathbf{1}, \mathbf{1} ; \mathbf{1})_{(-1 / 2,-2 / 27)}$ & $s$ \\
\hline 2 & $(\mathbf{1}, \mathbf{1} ; \mathbf{1})_{(1 / 2,-2)}$ & & 2 & $(\mathbf{1}, \mathbf{1} ; \mathbf{1})_{(-1 / 2,8)}$ & \\
\hline 1 & $(\mathbf{1}, \mathbf{1} ; \mathbf{1})_{(1 / 2,0)}$ & & 1 & $(\mathbf{1}, \mathbf{1} ; \mathbf{1})_{(-1 / 2,0)}$ & \\
\hline 1 & $(\mathbf{1}, \mathbf{1} ; \mathbf{1})_{(1 / 2,1 / 31)}$ & & 1 & $(\mathbf{1}, \mathbf{1} ; \mathbf{1})_{(-1 / 2,-1 / 31)}$ & \\
\hline 1 & $(\mathbf{1}, \mathbf{1} ; \mathbf{1})_{(1 / 2,-1 / 2)}$ & & 1 & $(\mathbf{1}, \mathbf{1} ; \mathbf{1})_{(-1 / 2,1 / 2)}$ & \\
\hline 1 & $(\mathbf{1}, \mathbf{1} ; \mathbf{1})_{(1 / 2,2)}$ & & 1 & $(\mathbf{1}, \mathbf{1} ; \mathbf{1})_{(-1 / 2,-2)}$ & \\
\hline 2 & $(\mathbf{1}, \mathbf{2} ; \mathbf{1})_{(0,0)}$ & $m$ & & & \\
\hline 2 & $(\mathbf{1}, \mathbf{2} ; \mathbf{1})_{(0,3 / 31)}$ & $m^{\prime}$ & 2 & $(\mathbf{1}, \mathbf{2} ; \mathbf{1})_{(0,-3 / 31)}$ & $\bar{m}^{\prime}$ \\
\hline 1 & $(\mathbf{1}, \mathbf{2} ; \mathbf{1})_{(0,1)}$ & & 1 & $(\mathbf{1}, \mathbf{2} ; \mathbf{1})_{(0,-1)}$ & \\
\hline 1 & $(\mathbf{1}, \mathbf{2} ; \mathbf{1})_{(0,-3)}$ & & 1 & $(\mathbf{1}, \mathbf{2} ; \mathbf{1})_{(0,3)}$ & \\
\hline
\end{tabular}

\begin{tabular}{|c|c|c|}
\hline$\#$ & Irrep & \\
\hline 11 & $(\mathbf{1}, \mathbf{1} ; \mathbf{1})_{(0,0)}$ & $s^{0}$ \\
\hline 4 & $(\mathbf{1}, \mathbf{1} ; \mathbf{1})_{(0, \pm 6)}$ & $\chi$ \\
\hline 6 & $(\mathbf{1}, \mathbf{1} ; \mathbf{1})_{(0,3)}$ & $\bar{\nu}$ \\
\hline$\overline{4}$ & $(\mathbf{1}, \mathbf{1} ; \mathbf{1})_{(0, \pm 21 / 31)}$ & $\tilde{s}$ \\
\hline 4 & $(\mathbf{1}, \mathbf{1} ; \mathbf{1})_{(0, \pm 1 / 2)}$ & \\
\hline 4 & $(\mathbf{1}, \mathbf{1} ; \mathbf{1})(0, \pm 3 / 2)$ & \\
\hline 2 & $(\mathbf{1}, \mathbf{1} ; \mathbf{1})_{(0, \pm 7 / 2)}$ & \\
\hline 2 & $(\mathbf{1}, \mathbf{1} ; \mathbf{1})_{(0, \pm 2 / 9)}$ & \\
\hline 2 & $(\mathbf{1}, \mathbf{1} ; \mathbf{1})_{(0, \pm 7 / 9)}$ & \\
\hline 2 & $(\mathbf{1}, \mathbf{1} ; \mathbf{1})_{(0, \pm 52 / 27)}$ & \\
\hline 2 & $(\mathbf{1}, \mathbf{1} ; \mathbf{1})_{(0, \pm 31 / 27)}$ & \\
\hline 2 & $(\mathbf{1}, \mathbf{1} ; \mathbf{1})_{(0, \pm 22 / 27)}$ & \\
\hline 2 & $(\mathbf{1}, \mathbf{1} ; \mathbf{1})_{(0, \pm 14 / 27)}$ & \\
\hline 2 & $(\mathbf{1}, \mathbf{1} ; \mathbf{1})_{(0, \pm 2 / 27)}$ & \\
\hline 2 & $(\mathbf{1}, \mathbf{1} ; \mathbf{1})_{(0, \pm 125 / 27)}$ & \\
\hline 2 & $(\mathbf{1}, \mathbf{1} ; \mathbf{1})_{(0, \pm 244 / 27)}$ & \\
\hline 2 & $(\mathbf{1}, \mathbf{1} ; \mathbf{1})_{(0, \pm 8 / 31)}$ & \\
\hline 2 & $(\mathbf{1}, \mathbf{1} ; \mathbf{1})_{(0, \pm 1 / 31)}$ & \\
\hline 2 & $(\mathbf{1}, \mathbf{1} ; \mathbf{1})(0, \pm 16 / 31)$ & \\
\hline 1 & $(\mathbf{1}, \mathbf{1} ; \mathbf{1})_{(0,-34 / 9)}$ & \\
\hline 1 & $(\mathbf{1}, \mathbf{1} ; \mathbf{1})_{(0,-20 / 9)}$ & \\
\hline 1 & $(\mathbf{1}, \mathbf{1} ; \mathbf{1})_{(0,29 / 3)}$ & \\
\hline 1 & $(\mathbf{1}, \mathbf{1} ; \mathbf{1})_{(0,-11 / 3)}$ & \\
\hline$\overline{2}$ & $(\mathbf{1}, \mathbf{1} ; \mathbf{6})_{(0,0)}$ & $x$ \\
\hline 2 & $(\mathbf{1}, \mathbf{1} ; \mathbf{6})_{(0, \pm 1 / 2)}$ & $x^{\prime}$ \\
\hline 2 & $(\mathbf{1}, \mathbf{1} ; \overline{\mathbf{6}})_{(0,0)}$ & $\bar{x}$ \\
\hline 2 & $(\mathbf{1}, \mathbf{1} ; \overline{\mathbf{6}})_{(0, \pm 1 / 2)}$ & $\bar{x}^{\prime}$ \\
\hline
\end{tabular}

Table 3: Massless spectrum of a model with $P_{6}$ as accidental symmetry. Quantum numbers w.r.t.

$\left[\mathrm{SU}(3)_{C} \times \mathrm{SU}(2)_{\mathrm{L}}\right] \times[\mathrm{SU}(6)]\left(\right.$ bold) and $\mathrm{U}(1)_{Y} \times \mathrm{U}(1)_{P_{6}}$ (subscripts) are given. 


\section{References}

[1] G. R. Farrar and P. Fayet, Phys. Lett. B76 (1978), 575-579.

[2] S. Dimopoulos and H. Georgi, Nucl. Phys. B193 (1981), 150.

[3] S. Dimopoulos, S. Raby, and F. Wilczek, Phys. Lett. B112 (1982), 133.

[4] L. E. Ibáñez and G. G. Ross, Phys. Lett. B260 (1991), 291-295.

[5] L. E. Ibáñez and G. G. Ross, Nucl. Phys. B368 (1992), 3-37.

[6] H. K. Dreiner, C. Luhn, and M. Thormeier, Phys. Rev. D73 (2006), 075007, hep-ph/0512163.

[7] C. Luhn and M. Thormeier, Phys. Rev. D77 (2008), 056002, [0711.0756].

[8] W. Buchmüller, K. Hamaguchi, O. Lebedev, and M. Ratz, Phys. Rev. Lett. 96 (2006), 121602, |hep-ph/0511035.

[9] W. Buchmüller, K. Hamaguchi, O. Lebedev, and M. Ratz, Nucl. Phys. B785 (2007), 149-209, hep-th/0606187.

[10] O. Lebedev et al., Phys. Lett. B645 (2007), 88-94, |hep-th/0611095].

[11] O. Lebedev et al., Phys. Rev. D77 (2008), 046013, [0708.2691].

[12] O. Lebedev, H. P. Nilles, S. Ramos-Sánchez, M. Ratz, and P. K. S. Vaudrevange, Phys. Lett. B668 (2008), 331-335, [0807.4384].

[13] T. Kobayashi, S. Raby, and R.-J. Zhang, Nucl. Phys. B704 (2005), 3-55, hep-ph/0409098.

[14] S. Förste, H. P. Nilles, P. K. S. Vaudrevange, and A. Wingerter, Phys. Rev. D70 (2004), 106008, [hep-th/0406208].

[15] T. Kobayashi, H. P. Nilles, F. Plöger, S. Raby, and M. Ratz, Nucl. Phys. B768 (2007), 135-156, hep-ph/0611020.

[16] T. Araki et al., Nucl. Phys. B805 (2008), 124-147, [0805.0207].

[17] B. Petersen, M. Ratz, and R. Schieren, JHEP 08 (2009), 111, [0907.4049].

[18] P. Ko, T. Kobayashi, J.-h. Park, and S. Raby, Phys. Rev. D76 (2007), 035005, [0704.2807].

[19] W. Buchmüller and J. Schmidt, Nucl. Phys. B807 (2009), 265-289, [0807.1046].

[20] O. Lebedev and S. Ramos-Sánchez, Phys. Lett. B684 (2010), 48-51, [0912.0477]. 
[21] H. Ishimori, T. Kobayashi, H. Ohki, H. Okada, Y. Shimizu and M. Tanimoto, Prog. Theor. Phys. Suppl. 183 (2010) 1, [1003.3552].

[22] R. Kappl, B. Petersen, M. Ratz, R. Schieren, and P. K. S. Vaudrevange, in preparation.

[23] I. Hinchliffe and T. Kaeding, Phys. Rev. D47 (1993), 279-284.

[24] S. P. Martin, (1997), hep-ph/9709356.

[25] Y. Grossman and H. E. Haber, Phys. Rev. D59 (1999), 093008, hep-ph/9810536.

[26] K. Hamaguchi, Y. Nomura, and T. Yanagida, Phys. Rev. D58 (1998), 103503, |hep-ph/9805346.

[27] K. S. Babu, I. Gogoladze, and K. Wang, Nucl. Phys. B660 (2003), 322-342, hep-ph/0212245.

[28] K. S. Babu, I. Gogoladze, and K. Wang, Phys. Lett. B570 (2003), 32-38, hep-ph/0306003.

[29] K. Wang, (2004), hep-ph/0407234.

[30] R. N. Mohapatra and M. Ratz, Phys. Rev. D76 (2007), 095003, [0707.4070].

[31] R. Slansky, Phys. Rept. 79 (1981), 1-128.

[32] H. P. Nilles, S. Ramos-Sánchez, M. Ratz, and P. K. S. Vaudrevange, Eur. Phys. J. C59 (2009), 249-267, [0806.3905].

[33] H. P. Nilles, S. Ramos-Sánchez, and P. K. S. Vaudrevange, AIP Conf. Proc. 1200 (2010), 226-234, [0909.3948].

[34] A. Font, L. E. Ibáñez, and F. Quevedo, Phys. Lett. B217 (1989), 272.

[35] T. Kobayashi, S. Raby, and R.-J. Zhang, Phys. Lett. B593 (2004), 262-270, hep-ph/0403065.

[36] J. Erler, J. Math. Phys. 35 (1994), 1819-1833, |hep-th/9304104].

[37] S. Stieberger, Nucl. Phys. B541 (1999), 109-144, hep-th/9807124.

[38] G. Honecker and M. Trapletti, (2006), hep-th/0612030.

[39] K.-S. Choi, I.-W. Kim, and J. E. Kim, JHEP 03 (2007), 116, hep-ph/0612107.

[40] K.-S. Choi, H. P. Nilles, S. Ramos-Sánchez, and P. K. S. Vaudrevange, Phys. Lett. B675 (2009), 381, [0902.3070]. 